\title{
Impact of Type 2 Diabetes on the Association Between Neurodegenerative Diseases and Cancer in Elderly Koreans: A 10-year Retrospective Cohort Study Using the National Health Insurance Service Database
}

Hee-Cheol Kim ( $\square$ mdhckim@gmail.com )

Keimyung University School of Medicine https://orcid.org/0000-0001-7957-4872

Ji-Hun Kim

Keimyung University School of Medicine

Ho-Jun Lee

Keimyung University School of Medicine

Yang-Tae Kim

Keimyung University School of Medicine

Byeong-Churl Jang

Keimyung University School of Medicine

\section{Research Article}

Keywords: Neurodegenerative disease, Cancer, Type 2 Diabetes, Cohort study, Epidemiology

Posted Date: September 23rd, 2021

DOI: https://doi.org/10.21203/rs.3.rs-844820/v2

License: (c) (i) This work is licensed under a Creative Commons Attribution 4.0 International License.

Read Full License 


\section{Abstract}

\section{Background}

Some studies suggest that cancer and type 2 diabetes (T2D), as well as neurodegenerative diseases (NDs), are related to protein misfolding and possibly to each other. This study aimed to investigate the association between NDs and cancer, and to determine the impact of T2D on the association between NDs and cancer.

\section{Methods}

A population-based 10-year follow-up study was conducted using the Korean National Health Insurance Service Database between 2002 and 2015. The study population included an ND group $(n=9,292)$ and control group ( $n=46,742)$, aged $\geq 60$ years. A multivariable Cox proportional hazards regression model was used to evaluate the hazard ratio (HR) and 95\% confidence interval (Cl) for cancer risk.

\section{Results}

Various another NDs and/or T2D were newly diagnosed in ND and control groups during the 10-year follow-up periods. Newly developed another ND cases before cancer onset were $2,412(26.0 \%)$ in the ND group and 15,435 (33.0\%) in the control group $(\chi 2=178.19, p<0.01)$. Newly developed T2D cases before cancer onset were 4,691 (50.5\%) and 25,695 (55.0\%) in the ND and control groups, respectively ( $\chi 2=$ $62.89, p<0.01$ ). As of December 31, 2015 (the end of the observation period), the number of cancer patients in the ND group was 2,040 (22.0\%) and that in the control group was 8,628 (18.5\%) $(x 2=61.45, p$ $<0.01)$. The ND group was associated with an increased risk of cancer $(\mathrm{HR}, 1.25 ; 95 \% \mathrm{Cl}, 1.19-1.31)$. In both groups, newly diagnosed another NDs and T2D showed an additive effect on cancer incidence during the follow-up period. The additive effect on cancer incidence was higher in T2D than in ND.

\section{Conclusion}

This study showed a positive association between NDs and cancer, and an additive effect of T2D on this association. Further replication studies are needed to address the association among NDs, cancer, and T2D.

\section{Introduction}

Neurodegenerative diseases (NDs) and cancer are often thought of as disease mechanisms at opposite ends of a spectrum; one due to premature cell death and the other due to enhanced resistance to cell death. There is now accumulating evidence to link these two disparate processes and they share common mechanisms of genetic and molecular abnormalities [1-3]. Several NDs are classified as protein misfolding diseases, as they are associated with misfolded protein aggregation. Recent research suggests that aggregated proteins, the longtime hallmarks of NDs, also play a role in cancer $[4,5]$. In addition to NDs and cancer, type 2 diabetes (T2D), a common disease in the elderly, can be classified as a 
protein misfolding disorder. Recent evidence suggests that formation of toxic aggregates of the islet amyloid polypeptide (IAPP) might contribute to $\beta$-cell dysfunction and disease [6]. The presence of misfolded IAPP aggregates of different sizes ranging from small soluble oligomers to large fibrillar aggregates deposited in the Islets of Langerhans of patients affected by T2D is well established. However, the mechanism of protein aggregation and its associated toxicity is still unclear [7].

A growing body of evidence links T2D with NDs such as Alzheimer's disease (AD). Epidemiological studies show that T2D increases the risk of $A D[8,9]$. Moreover, other epidemiological studies have suggested that T2D appear to be associated with cancers, particularly hepatic, pancreatic, colorectal, bladder, endometrial, and breast cancers [10-15]. Several biological mechanisms have been suggested to explain the link between T2D and cancer $[16,17]$. In addition to the association between T2D and cancer, previous studies have reported on the association between ND and cancer. Many epidemiological studies show that NDs and cancer are inversely related; for instance, a lower incidence of AD dementia in people with cancer, and less cancer in persons with $A D$ dementia [18-20]. Contrarily, in the cases of research on the relationship between Parkinson's disease (PD) and cancer, results from previous epidemiological studies are inconsistent. Many epidemiological studies have reported associations between PD and cancers, which is supporting a general inverse association and more recently, a positive association in certain cancers including skin, breast, and brain [21]. Some studies have reported that patients with PD have a lower risk of developing cancer [22], but other studies have reported the opposite [23].

Several previous studies suggest that cancer and T2D, as well as NDs, are related to protein misfolding, and these diseases may be related to each other. This study aimed to determine whether these diseases are associated with each other. For this purpose, we conducted this nationwide population-based, ageand sex-matched cohort study to investigate cancer incidence in patients with various NDs and without any NDs, using the National Health Insurance Service (NHIS) database (DB) for the entire Korean population. We also investigated the impact of T2D on the association between NDs and cancer.

\section{Methods}

This study used data from the Korean NHIS DB. We obtained permission from the National Health Insurance Corporation's Review Committee to use the national health information data (NHIS-2021-1254). The study was conducted according to the ethical principles outlined in the Declaration of Helsinki. All study procedures and ethical aspects were approved by the Institutional Review Board (IRB) of Keimyung University Dongsan Hospital (IRB number: DSMC 2019-08-067-004).

\section{The NHIS Database}

All Korean citizens are required to enroll in the Korean NHIS. A total of $97 \%$ and $3 \%$ of the Korean population is covered by the Medical Assistance Program and the Medical Care for Patriots and Veterans Affairs Scheme, respectively. The data collected by the NHIS DB include detailed treatment practices and prescriptions based on the fee-for-service payment model, as well as the medical information of all 
citizens who signed up for medical insurance in Korea. Data include insurance eligibility and premiums from birth to death of all citizens, hospital medical history and national health examination results, rare refractory and cancer registration information, medical benefits data, and elderly long-term care data since 2002. The NHIS claims for inpatient and outpatient visits, procedures, and prescriptions are coded using the International Classification of Diseases, 10th Revision (ICD-10), adopted in Korea in 1995, and the Korean Drug and Anatomical Therapeutic Chemical Codes [24]. The NHIS routinely audits the claims, and the data are considered reliable and used in numerous peer-reviewed publications $[25,26]$.

The data sharing service of the NHIS DB is largely divided into customized and sample DBs. The customized DB (NHIS-National Health Information Database; NHIS-NHID) is representative of the transmission data provided by de-identifying health insurance and long-term care insurance data collected by the DB. As the data are cohort-based, longitudinal studies are possible, as are studies on low prevalence diseases including rare ones using a customized database. This DB can be used for a population-based cohort study, according to the operational definition of some diseases $[27,28]$. We used a customized DB to select the study population suitable for the research purpose.

\section{Study Population}

This study used NHIS-Customized Data (NHIS-2021-1-254) for 14 years from 2002 to 2015 including NHIS's health insurance and long-term care insurance data for the entire population of Korea. In this study, to select patients without both NDs and cancer before study initiation, the 1-year period prior to 2003 was set as a "wash-out" period. We only extracted data from patients aged $\geq 60$ years as of 2003 among patients who had no diagnostic codes for cancer and any NDs in 2002. Diagnoses in the NHIS DB are based on the Korean Classification of Disease, 6th version (KCD-6), which is essentially a revision of the ICD-10. Diagnoses obtained from medical insurance claims data may be inaccurate compared with diagnoses obtained from medical charts. Therefore, to increase the diagnostic accuracy, patients were regarded as having ND if they had $\geq 1$ medical claim with a diagnostic code of AD (KCD-6 codes G300, G301, G308, G309, F000, F001, F002, or F009), PD (KCD-6 codes G20 or F023), or circumscribed brain atrophy (CBA) (KCD-6 codes G310, G3101, G3102, G3103, G3104, G3108, or F020) and had received prescriptions for antidementia drugs (donepezil, rivastigmine, memantine, or galantamine) and/or antiparkinsonian drugs $\geq 1$ times after their first-time diagnosis between January 2003 and December 2005. Cancer was determined from a diagnosis code of malignant neoplasms (C00-C97), in situ neoplasms (D00-D09), and neoplasms of uncertain or unknown behavior (D37-D48). T2D was defined if KCD- 6 codes with E11 were assigned $\geq 2$ times per patient. Patients with any diagnostic code of NDs and cancer before 2003 or those with a diagnostic code of vascular dementia (KCD-6 codes F010, F011, F012, F013, F018, and F019) were excluded.

Among the data extracted through this process, during the study enrollment period, 2003-2005, patients with ND diagnosis for the first time who have never diagnosed with cancer (ND group, 9,292 patients) and patients without both ND and cancer diagnoses (non-ND group, 618,112 patients) were re-extracted. The ND group was composed of 3,141 AD patients, 5,812 PD patients, and 339 CBA patients. The CBA patients include frontotemporal dementia, Pick disease, and progressive isolated aphasia. Among the 
non-ND group, patients with similar characteristics to the AD group, including age, sex, and comorbid diseases were extracted. We used 1:5 propensity score matching (PSM) method to match patients of nonND group to patients of ND group. 46,742 pairs (7.6\% of patients of non-ND group) were matched for control group. Propensity scores were calculated based on the subjects' age, sex, and comorbidity assessed using the Charlson Comorbidity Index (CCl)-total score. Therefore, the total number of patients included in this study was 56,034, including 9,292 patients in the ND group and 46,742 patients from the control group. Based on the patient data extracted through this process, these patients were followed up for 10 years from January 1, 2006 to December 31, 2015, and the incidence of cancer was investigated.

\section{Data Management and Statistical Analysis}

Stata/MP 16 (Stata Corp LLC, College Station, TX, USA) was used for data management and statistical analysis [29]. Data are presented as mean \pm standard deviation for continuous variables and number with percentages for categorical variables. Comparison between the ND and control groups for continuous variables was conducted by Student t-test. Chi-square test was performed to determine the difference in the cancer frequency between the ND and control groups. The Kaplan-Meier method was used to estimate the risk for developing cancer over the 10-year follow-up period, and the log-rank test was used to examine differences in cancer incidence between the ND and control groups. Multivariable Cox proportional hazards model was used to estimate the adjusted hazard ratio (HR) and $95 \%$ confidence intervals (Cls) for cancer incidence after adjusting for confounding comorbidities, age, and sex. All statistical tests were two-sided, and $P$ values less than 0.05 were considered as statistically significant.

\section{Results}

\section{Sample Characteristics}

Before the PSM, there were statistically significant differences in sex, age, and CCl-total scores between the ND and control groups, but after the PSM, the difference between the two groups was no longer present except for age, and the matching was relatively good (Table 1). Although the matching method was used for the case-control study, there was a significant difference between the ND and control groups in the frequency of baseline T2D at the beginning of the study $(27.7 \%$ vs. $20.0 \%$, respectively; $\chi 2=$ $270.33, p<0.01)$. 
Table 1

Characteristics of study subjects before and after propensity score matching (PSM)

\begin{tabular}{|lllllll|}
\hline Variables & Before PSM & \multicolumn{5}{c|}{ After PSM } \\
\cline { 2 - 7 } & Control group & ND group & $\boldsymbol{p}$ & Control group & ND group & $\boldsymbol{p}$ \\
\hline Sex & $281,093(45.5)$ & $2,209(23.8)$ & $<0.01$ & $11,178(23.9)$ & $2,209(23.8)$ & 0.77 \\
Male (\%) & $337,019(54.5)$ & $7,083(76.2)$ & & $35,564(76.1)$ & $7,083(76.2)$ & \\
Female (\%) & & & & & & \\
\hline Age & $70.21 \pm 4.91$ & $71.02 \pm 4.89$ & $<0.01$ & $71.86 \pm 4.62$ & $71.02 \pm 4.89$ & $<0.01$ \\
\hline CCl total & $0.25 \pm 0.56$ & $0.47 \pm 0.75$ & $<0.01$ & $0.48 \pm 0.76$ & $0.47 \pm 0.75$ & 0.07 \\
\hline ND Neurodegenerative disease, CC/Charlson comorbidity index & & \\
\hline
\end{tabular}

\section{Frequency of Newly Diagnosed ND and T2D}

The subjects of both the ND and control groups were patients not diagnosed with cancer or ND before 2003, and those in the ND group were diagnosed with ND for the first time during the enrollment period, from 2003 to 2005. However, these subjects continued to develop new or another NDs before cancer onset over a 10-years follow-up period from 2006 to 2015. Therefore, we investigated cases of newly diagnosed ND before cancer onset between 2006 and 2015 in the ND and control groups. In this case, the diagnostic criterion for various NDs was set as cases with a diagnostic code for the disease in the main diagnosis or auxiliary diagnosis (including all first to third auxiliary diagnosis) and antidementia and/or antiparkinsonian drugs administration not considered as diagnostic criterion. Among all cases, 2,412 $(26.0 \%)$ cases in the ND group and 15,435 (33.0\%) in the control group were diagnosed with another ND (in the ND group) or new ND (in the control group) before cancer onset over a 10-years follow-up period $(x 2=178.19, p<0.01)$.

Among the total study population, there were some patients with baseline T2D at the time of study enrollment, and patients with newly diagnosed T2D during the follow-up period. The number of patients with baseline T2D was 2,572 (27.7\%) in the ND group and 9,364 (20.0\%) in the control group $(x 2=270.33$, $p<0.01)$. The number of patients with newly diagnosed T2D before cancer onset during the follow-up period was 4,691 (50.5\%) in the ND group and 25,695 (55.0\%) in the control group $(\chi 2=62.89, p<0.01)$.

\section{Incidence of Cancer}

As of December 31, 2015 (the end of the observation period), the number of cancer patients in the ND group was 2,040 (22.0\%) and that in the control group was 8,628 $(18.5 \%)(\chi 2=61.45, p<0.01)$. The incidence of cancer was significantly higher in the ND group than in the control. Since the length from the observation period to cancer diagnosis differed among patients, the cancer incidence rates were calculated per 1,000 person-years in this study. The cancer incidence rate was 25.3 per 1,000 personyears $(95 \% \mathrm{Cl}, 24.3 \sim 26.5)$ in the ND group and 20.4 per 1,000 person-years $(95 \% \mathrm{Cl}, 20.0 \sim 20.9)$ in the control group. 
In this study, new NDs not existing at the time of study enrollment occurred in the control group and ND group during the follow-up period prior to cancer onset. Therefore, to determine the effect of newly diagnosed ND on cancer onset during the follow-up period, the relationship between the frequency of newly diagnosed ND and cancer during the follow-up period was investigated. The number of cancer patients was 3,459 (19.4\%) in total patients with newly diagnosed ND and 7,209 (18.9\%) in total patients without newly diagnosed ND, and there was no significant difference between them $(\chi 2=2.00, p=0.16)$. However, when analyzed by separating these total patients into the ND and control groups, the incidence of cancer was significantly higher in patients with newly diagnosed ND within the ND group. In the control group, there was still no significant difference between patients with and without newly diagnosed ND (Table 2). 
Table 2

Comparison of the cancer frequency at the end of the follow-up period

\begin{tabular}{|c|c|c|c|c|}
\hline Group & Cancer (\%) & No cancer $(\%)$ & $x^{2}$ & $p$ \\
\hline \multicolumn{5}{|l|}{ Total population } \\
\hline Newly diagnosed ND & $3,459(19.4)$ & $14,388(80.6)$ & 2.00 & 0.16 \\
\hline \multicolumn{5}{|l|}{ Yes } \\
\hline No & 7,209 (18.9) & $30,978(81.1)$ & & \\
\hline Newly diagnosed T2D & 6,459 (21.3) & $23,927(78.7)$ & 211.89 & $<0.01$ \\
\hline \multicolumn{5}{|l|}{ Yes } \\
\hline NO & $4,209(16.4)$ & 21,439 (83.6) & & \\
\hline \multicolumn{5}{|l|}{ Control group } \\
\hline Newly diagnosed ND & 2,891 (18.7) & $12,544(81.3)$ & 1.13 & 0.29 \\
\hline \multicolumn{5}{|l|}{ Yes } \\
\hline No & $5,737(18.3)$ & $25,570(81.7)$ & & \\
\hline Newly diagnosed T2D & $5,279(20.5)$ & $20,416(79.5)$ & 164.99 & $<0.01$ \\
\hline \multicolumn{5}{|l|}{ Yes } \\
\hline No & $3,349(15.9)$ & 17,698 (84.1) & & \\
\hline \multicolumn{5}{|l|}{ ND group } \\
\hline Newly diagnosed ND & $568(23.5)$ & $1,844(76.5)$ & 4.83 & 0.03 \\
\hline \multicolumn{5}{|l|}{ Yes } \\
\hline No & $1,472(21.4)$ & $5,408(78.6)$ & & \\
\hline Newly diagnosed T2D & $1,180(25.2)$ & $3,511(74.8)$ & 56.62 & $<0.01$ \\
\hline \multicolumn{5}{|l|}{ Yes } \\
\hline No & $860(18.7)$ & $3,741(81.3)$ & & \\
\hline
\end{tabular}

The effect of newly diagnosed T2D on cancer onset during the follow-up period was also investigated. There was a significant difference between the number of cancer patients in total patients with newly diagnosed T2D, which was 6,459 (21.3\%) cases, and 4,209 cases (16.4\%) in all patients without newly diagnosed T2D $(x 2=211.89, p<0.01)$. The results did not change even when the groups were split into the ND and control groups (Table 2). 
Because cancer did not develop in all patients in the study during the follow-up period, the cancer-free average survival time can only be evaluated by limiting the maximum observation period to cancer onset (restricted mean survival time). The restricted mean survival time was 8.66 years $(95 \% \mathrm{Cl}, 8.61-8.72)$ in the ND group and 9.04 years $(95 \% \mathrm{Cl}, 9.02-9.06)$ in the control group. The log-rank test showed a significantly different pattern of cumulative cancer-free survival probabilities between the two groups ( $\chi 2$ $=60.48, p<0.01)$. To evaluate the impact of newly diagnosed ND and T2D on the incidence of cancer between the ND and control groups, we categorized subjects into eight groups according to the presence or absence of newly diagnosed ND and/or T2D. Group 1 was a control group without both newly diagnosed ND and T2D ( $n=14,825,26.5 \%)$; Group 2, a control group with only newly diagnosed ND (no T2D) $(n=6,222,11.1 \%)$; Group 3, a control group with only newly diagnosed T2D (no ND) ( $n=16,482$, 29.4\%); Group 4, a control group with both newly diagnosed ND and T2D ( $n=9,213,16.4 \%)$; Group 5, an ND group without both newly diagnosed ND and T2D ( $n=3,499,6.2 \%)$; Group 6, an ND group with only newly diagnosed ND (no T2D) ( $n=1,102,2.0 \%$ ); Group 7, an ND group with only newly diagnosed T2D (no ND) $(n=3,381,6.0 \%)$, and Group 8, an ND group with both newly diagnosed ND and T2D $(n=1,310$, $2.3 \%$ ). Figure 1 shows the Kaplan-Meier survival curves of the eight groups. The frequencies and incidence rates (per 1,000 person-years) of cancer over a 10-year follow-up period are presented in Table 3. After adjusting for age, sex, and comorbidities (CCl-total score), the adjusted HR for cancer in patients of ND group during the 10-year follow-up period was $1.25(95 \% \mathrm{Cl}, 1.19 \sim 1.31$; $\mathrm{P}<0.01)$ compared with the HRs of the patients in the control group. Table 4 shows the comparison of adjusted HRs for cancer in two out of the eight groups. In both the control group and ND group, the adjusted HR for cancer gradually increased in the following order: cases without both newly diagnosed ND and T2D, cases with only newly diagnosed ND, cases with only newly diagnosed T2D, and cases with both newly diagnosed ND and T2D. 
Table 3

Frequencies and incidence rates of cancer over a 10-year follow-up period

\begin{tabular}{|c|c|c|c|c|}
\hline Group & Person-years & No. of cancer & $\mathbf{I R}$ & $95 \% \mathrm{Cl}$ \\
\hline Group 1 & $136,304.87$ & 2,324 & 17.05 & $16.37 \sim 17.76$ \\
\hline Group 2 & $56,625.51$ & 1,025 & 18.10 & $17.03 \sim 19.24$ \\
\hline Group 3 & $147,433.41$ & 3,413 & 23.15 & $22.39 \sim 23.94$ \\
\hline Group 4 & $82,164.74$ & 1,866 & 22.71 & $21.70 \sim 23.76$ \\
\hline Group 5 & $31,109.90$ & 631 & 20.28 & $18.76 \sim 21.93$ \\
\hline Group 6 & $9,585.62$ & 229 & 23.89 & $20.99 \sim 27.19$ \\
\hline Group 7 & $28,853.03$ & 841 & 29.15 & $27.24 \sim 31.19$ \\
\hline Group 8 & $10,962.94$ & 339 & 30.92 & $27.80 \sim 34.40$ \\
\hline \multicolumn{5}{|c|}{$I R$ Incidence rate (per 1000 person-years), $C /$ Confidence interval } \\
\hline \multicolumn{5}{|c|}{$\begin{array}{l}\text { Group } 1 \text { is a control group without both newly diagnosed ND and T2D }(n=14,825,26.5 \%) \text {, Group } 2 \text { is } \\
\text { a control group with only newly diagnosed ND (no T2D) }(n=6,222,11.1 \%) \text {, Group } 3 \text { is a control group } \\
\text { with only newly diagnosed T2D (no ND) ( } n=16,482,29.4 \%) \text {, Group } 4 \text { is a control group with both } \\
\text { newly diagnosed ND and T2D ( } n=9,213,16.4 \%) \text {, Group } 5 \text { is an ND group without both newly } \\
\text { diagnosed ND and T2D ( } n=3,499,62 \%) \text {, Group } 6 \text { is an ND group with only newly diagnosed ND (no } \\
\text { T2D) ( } n=1,102,2.0 \% \text { ), Group } 7 \text { is an ND group with only newly diagnosed T2D (no ND) ( } n=3,381 \text {, } \\
6.0 \%) \text {, Group } 8 \text { is an ND group with both newly diagnosed ND and T2D ( }=1,310,2.3 \% \text { ) }\end{array}$} \\
\hline
\end{tabular}


Table 4

Adjusted hazard ratios and $95 \%$ confidence interval for cancer

\begin{tabular}{|c|c|c|c|c|c|c|c|c|}
\hline & Group 1 & Group 2 & Group 3 & Group 4 & Group 5 & Group 6 & Group 7 & Group 8 \\
\hline \multirow{2}{*}{$\begin{array}{l}\text { Group } \\
1\end{array}$} & & 1.18 & 1.35 & 1.47 & 1.23 & 1.47 & 1.74 & 1.87 \\
\hline & & $\begin{array}{l}(1.09 \sim \\
1.27)\end{array}$ & $\begin{array}{l}(1.28 \sim \\
1.43)\end{array}$ & $\begin{array}{l}(1.38 \sim \\
1.56)\end{array}$ & $\begin{array}{l}(1.12 \sim \\
1.34)\end{array}$ & $\begin{array}{l}(1.29 \sim \\
1.69)\end{array}$ & $\begin{array}{l}(1.61 \sim \\
1.88)\end{array}$ & $\begin{array}{l}(1.67 \sim \\
2.10)\end{array}$ \\
\hline \multirow{2}{*}{$\begin{array}{l}\text { Group } \\
2\end{array}$} & 1.17 & & 1.15 & 1.25 & 1.04 & 1.26 & 1.48 & 1.59 \\
\hline & $\begin{array}{l}(1.09 \sim \\
1.26)\end{array}$ & & $\begin{array}{l}(1.07 \sim \\
1.24)\end{array}$ & $\begin{array}{l}(1.15 \sim \\
1.35)\end{array}$ & $\begin{array}{l}(0.94 \sim \\
1.15)\end{array}$ & $\begin{array}{l}(1.09 \sim \\
1.45)\end{array}$ & $\begin{array}{l}(1.35 \sim \\
1.62)\end{array}$ & $\begin{array}{l}(1.41 \sim \\
1.80)\end{array}$ \\
\hline \multirow{2}{*}{$\begin{array}{l}\text { Group } \\
3\end{array}$} & 1.39 & 1.19 & & 1.08 & 0.90 & 1.09 & 1.29 & 1.38 \\
\hline & $\begin{array}{l}(1.31 \sim \\
1.48)\end{array}$ & $\begin{array}{l}(1.10 \sim \\
1.28)\end{array}$ & & $\begin{array}{l}(1.02 \sim \\
1.15)\end{array}$ & $\begin{array}{l}(0.83 \sim \\
0.98)\end{array}$ & $\begin{array}{l}(0.95 \sim \\
1.25)\end{array}$ & $\begin{array}{l}(1.19 \sim \\
1.39)\end{array}$ & $\begin{array}{l}(1.24 \sim \\
1.54)\end{array}$ \\
\hline \multirow{2}{*}{$\begin{array}{l}\text { Group } \\
4\end{array}$} & 1.50 & 1.28 & 1.08 & & 0.84 & 1.01 & 1.19 & 1.28 \\
\hline & $\begin{array}{l}(1.40 \sim \\
1.61)\end{array}$ & $\begin{array}{l}(1.18 \sim \\
1.39)\end{array}$ & $\begin{array}{l}(1.00 \sim \\
1.16)\end{array}$ & & $\begin{array}{l}(0.76 \sim \\
0.92)\end{array}$ & $\begin{array}{l}(0.88 \sim \\
1.16)\end{array}$ & $\begin{array}{l}(1.09 \sim \\
1.29)\end{array}$ & $\begin{array}{l}(1.14 \sim \\
1.43)\end{array}$ \\
\hline \multirow{2}{*}{$\begin{array}{l}\text { Group } \\
5\end{array}$} & 1.23 & 1.05 & 0.88 & 0.82 & & 1.20 & 1.42 & 1.53 \\
\hline & $\begin{array}{l}(1.12 \sim \\
1.35)\end{array}$ & $\begin{array}{l}(0.94 \sim \\
1.16)\end{array}$ & $\begin{array}{l}(0.80 \sim \\
0.97)\end{array}$ & $\begin{array}{l}(0.74 \sim \\
0.91)\end{array}$ & & $\begin{array}{l}(1.04 \sim \\
1.40)\end{array}$ & $\begin{array}{l}(1.28 \sim \\
1.58)\end{array}$ & $\begin{array}{l}(1.34 \sim \\
1.74)\end{array}$ \\
\hline \multirow{2}{*}{$\begin{array}{l}\text { Group } \\
6\end{array}$} & 1.44 & 1.23 & 1.04 & 0.96 & 1.18 & & 1.18 & 1.27 \\
\hline & $\begin{array}{l}(1.24 \sim \\
1.68)\end{array}$ & $\begin{array}{l}(1.05 \sim \\
1.44)\end{array}$ & $\begin{array}{l}(0.89 \sim \\
1.20)\end{array}$ & $\begin{array}{l}(0.83 \sim \\
1.12)\end{array}$ & $\begin{array}{l}(1.00 \sim \\
1.39)\end{array}$ & & $\begin{array}{l}(1.02 \sim \\
1.37)\end{array}$ & $\begin{array}{l}(1.07 \sim \\
1.50)\end{array}$ \\
\hline \multirow{2}{*}{$\begin{array}{l}\text { Group } \\
7\end{array}$} & 1.78 & 1.52 & 1.28 & 1.19 & 1.45 & 1.23 & & 1.07 \\
\hline & $\begin{array}{l}(1.62 \sim \\
1.96)\end{array}$ & $\begin{array}{l}(1.37 \sim \\
1.69)\end{array}$ & $\begin{array}{l}(1.16 \sim \\
1.41)\end{array}$ & $\begin{array}{l}(1.07 \sim \\
1.32)\end{array}$ & $\begin{array}{l}(1.29 \sim \\
1.64)\end{array}$ & $\begin{array}{l}(1.04 \sim \\
1.46)\end{array}$ & & $\begin{array}{l}(0.95 \sim \\
1.22)\end{array}$ \\
\hline \multirow{2}{*}{$\begin{array}{l}\text { Group } \\
8\end{array}$} & 1.92 & 1.64 & 1.38 & 1.28 & 1.57 & 1.33 & 1.08 & \\
\hline & $\begin{array}{l}(1.67 \sim \\
2.22)\end{array}$ & $\begin{array}{l}(1.41 \sim \\
1.91)\end{array}$ & $\begin{array}{l}(1.20 \sim \\
1.59)\end{array}$ & $\begin{array}{l}(1.11 \sim \\
1.49)\end{array}$ & $\begin{array}{l}(1.34 \sim \\
1.84)\end{array}$ & $\begin{array}{l}(1.09 \sim \\
1.62)\end{array}$ & $\begin{array}{l}(0.92 \sim \\
1.27)\end{array}$ & \\
\hline \multicolumn{9}{|c|}{ ND Neurodegenerative disease, T2D Type 2 diabetes } \\
\hline \multicolumn{9}{|c|}{$\begin{array}{l}\text { Group } 1 \text { is a control group without both newly diagnosed ND and T2D, Group } 2 \text { is a control group with } \\
\text { only newly diagnosed ND (no T2D), Group } 3 \text { is a control group with only newly diagnosed T2D (no } \\
\text { ND), Group } 4 \text { is a control group with both newly diagnosed ND and T2D, Group } 5 \text { is an ND group } \\
\text { without both newly diagnosed ND and T2D, Group } 6 \text { is an ND group with only newly diagnosed ND } \\
\text { (no T2D), Group } 7 \text { is an ND group with only newly diagnosed T2D (no ND), Group } 8 \text { is an ND group } \\
\text { with both newly diagnosed ND and T2D }\end{array}$} \\
\hline
\end{tabular}

\section{Discussion}


This nationwide population-based cohort study demonstrated an $18.8 \%$ increase in total cancer risk among ND patients compared to control patients without ND over a 10-year follow-up period. The adjusted HR for cancer in patients of the ND group was $1.25(95 \% \mathrm{Cl}, 1.19 \sim 1.31 ; \mathrm{P}<0.01)$ compared with the HR in patients of control group. During the 10-year follow-up period, compared with the pure control group where no ND or T2D occurred, the cancer risk ratio of the ND group additionally diagnosed with T2D and other ND during the follow-up period increased to 1.87. These results suggest that the cancer risk is further increased as new ND and T2D were additionally diagnosed during the 10-year follow-up period. These findings are contrary to the results of previous epidemiologic studies examining the association between ND and cancer. This difference between the results of this study and previous ones is likely due to methodological differences. In many previous studies on the association between a particular ND (AD or PD) and cancer, the control group for ND was simply selected as subjects not having AD or PD. In such cases, NDs other than AD or PD may be included in the control group, which may affect cancer development in the control group. To confirm the selection bias in the process of control group selection, we separately analyzed only data of $A D$ patients, and cancer incidence rates were compared with two types of control groups for $A D$ (non-ND control group and non-AD control group). The number of cancer patients in $A D$ patients was $632 / 3,141$ (20.1\%). When the control group was selected as patients without any ND (non-ND control group), 8,628 (18.5\%) out of 46,742 patients in the non-ND control group were diagnosed with cancer $(\chi 2=5.38, p=0.02$, compared with $A D$ patients), but when the control group was selected as patients only without $A D$ (non-AD control group; in this case, ND other than $A D$ may be included in the control group), 10,036 (19.0\%) out of 52,893 patients in the non-AD control group were diagnosed with cancer $(X 2=2.53, p=0.11$, compared with AD patients). Therefore, this finding shows that cancer incidence slightly increases when NDs other than AD were included in the control group. These results indicate that the lower cancer incidence in the $A D$ group compared to the control group in previous epidemiologic studies could not completely rule out the effect on cancer incidence of included NDs other than $A D$ in the control group. In this study, patients without any ND were selected as a control group, and as a result, the incidence of cancer was higher in the ND group over a 10-year follow-up period compared to the control group.

During the 10-year follow-up period of this study, there were new cases of ND and/or T2D not present at the beginning of the study. These new ND and/or T2D cases may affect the development of subsequent cancers; therefore, we also investigated the impact of these new cases on the development of subsequent cancers. In both the control and ND groups, newly diagnosed ND and T2D showed an additive effect on the incidence of cancer during the follow-up period. The additive effect on cancer incidence was higher in T2D than in ND. This study showed a positive association between ND and cancer and that T2D had an additive effect on this association. These results are consistent with the fact that, unlike previously thought only in the aspect of apoptosis, recent molecular biology studies have suggested that the pathophysiology of cancer and NDs have a common mechanism. Signaling pathways, such as those arising from DNA damage, deviation from the normal cell cycle, inflammation, and oxidative stress that affect cell death and survival, have been studied in connection with cancer development, but recent studies have also linked them to NDs (1-3). p53, cyclin D, cyclin E, cyclin F, Pin1, and protein 
phosphatase 2A are commonly involved in the pathophysiology of cancer and NDs and are involved in cell cycle regulation [30,31]. Among these, $p 53$ is the most widely studied tumor suppressor gene and known to be associated with $50 \%$ of cancer [32]. It is well known that p53 plays an important role in apoptosis, and in most cancers, mutations at the gene level prevent it from functioning properly, resulting in poor activity of p53, which allows cancer cells to escape from apoptosis, leading to cancer. However, not only changes at the gene level, but the protein folding process is also an important factor in preventing p53 from normal functioning [33-35]. Cancer occurs due to not only a mutation in the p53 protein but also a problem in the p53 folding process [36]. In addition to the association between ND and cancer, many studies have reported that T2D is also associated with ND and cancer. In the relationship between T2D and ND, amylin's (or IAPP) functions in the periphery and its impact on pancreatic cell function and T2D progression have been described $[6,37]$. The presence of AD-related proteins ( $\beta$ amyloid protein and tau protein) in the pancreas and insulin-sensitive tissues and their roles in inducing peripheral insulin resistance or disruptions in insulin secretion are a potential mechanistic link among these AD-related proteins promoting T2D. Similarly, amylin accumulation in the brain, its ability to induce neurotoxicity, and form "cross-seeding" aggregates with $\beta$-amyloid protein illustrates the role for this pancreatic amyloidogenic protein in neurodegeneration $[38,39]$. The association between diabetes and cancer is well established [40,41]. Epidemiologic studies suggest that people with diabetes (predominantly T2D) are at a significantly higher risk for many forms of cancer [42, 43]. However, the risk of prostate cancer is lower in men with T2D [44]. The relationship between T2D and cancer varies depending on the cancer site, and more rigorous and collaborative studies are needed to understand the relationship between the two diseases.

Our study has some limitations. First, this study was a retrospective observational design based on claims data, and the diagnoses for NDs identified using KCD- 6 codes in the claims database, which may have been inaccurate compared with diagnoses obtained from a medical chart, neuroimaging tests, or neuropsychological tests. Second, this study did not analyze the association between NDs and various types of cancer. Third, patients with NDs tend to have less frequent contact with the healthcare system, including cancer examinations, which may have caused surveillance bias. We investigated the utilization rate of medical institutions in patients of the ND and control groups to examine the possibility of this surveillance bias. The annual average number of visits to medical institutions before cancer onset was investigated. There was no significant difference in the annual average number of visits to medical institutions between the two groups, with an average of $26.8 \pm 19.8$ in all patients in the ND group and an average of $26.6 \pm 18.7$ in all patients in the control group $(t=-0.75, p=0.45)$. Even when separately analyzing patients with cancer, there was no significant difference in the annual average number of visits to medical institutions between the two groups, with an average of $25.0 \pm 18.7$ in all patients in the ND group and an average of $24.8 \pm 16.7$ in all patients in the control group $(t=-0.55, p=0.58)$.

\section{Conclusion}

This study showed a positive association between NDs and cancer. Further, T2D diagnosed during the follow-up period was shown to have an additive effect on the association between NDs and cancer. 
These results show that the NDs, cancer, and T2D are related to each other and suggest that they may share a common pathophysiological process. Further replication studies are needed to address the association among the NDs, cancer, and T2D.

\section{Abbreviations}

AD: Alzheimer's disease; CBA; Circumscribed brain atrophy; CCl: Charlson Comorbidity Index; Cl:

Confidence interval; DB: Database; HR: Hazard ratio; IAPP: Islet amyloid polypeptide; ICD-10: International Classification of Diseases, $10^{\text {th }}$ revision; IRB: Institutional review board; KCD-6: Korean Classification of Disease, $6^{\text {th }}$ version; ND: Neurodegenerative disease; NHIS: National health insurance service; NHIS-NHID: NHIS-National Health Information Database; PD: Parkinson's disease; PSM: Propensity score matching; T2D: Type 2 diabetes

\section{Declarations}

\section{Authors' contributions}

H.C.K. conceived and designed the study; H.C.K. and J.H.K. analyzed the data: H.C.K., J.H.K., H.J.L, Y.T.K., and B.C.J. participated in the interpretation of data; H.C.K., B.C.J., and J.H.K. wrote and reviewed the manuscript. H.J.L. and Y.T.K. reviewed the manuscript. All authors have read and agreed to the published version of the manuscript.

\section{Funding}

This work was supported by the research promoting grant from the Keimyung University Dongsan Medical Center in 2020.

\section{Availability of data and materials}

The datasets used and/or analyzed during the current study are available from the corresponding author on reasonable request.

\section{Ethics approval and consent to participate}

This study used data from the Korean NHIS DB. We obtained approval from the National Health Insurance Corporation's Review Committee for permission to use of national health information data (NHIS-2021-1-254). The study was conducted according to the ethical principles outlined in the Declaration of Helsinki. All study procedures and ethical aspects were approved by the Institutional Review Board (IRB) of Keimyung University Dongsan Hospital (IRB number: DSMC 2019-08-067-004).

\section{Consent for publication}

Not applicable. 


\section{Competing interests}

The authors declare no financial interest or conflict of interest.

\section{Author details}

${ }^{1}$ Department of Psychiatry, Keimyung University School of Medicine, Daegu 42601, South Korea. ${ }^{2}$ Brain Research Institute, Keimyung University School of Medicine, Daegu 42601, South Korea. ${ }^{3}$ Department of Molecular Medicine, Keimyung University School of Medicine, Daegu 42601, South Korea.

\section{Acknowledgements}

Not applicable.

\section{ORCID iDs}

Hee-Cheol Kim https://orcid.org/0000-0001-7957-4872

Ji-Hun Kim https://orcid.org/0000-0003-2786-9737

Ho-Jun Lee https://orcid.org/0000-0001-8154-4373

Yang-Tae Kim https://orcid.org/0000-0002-1609-6807

Beong-Churl Jang https://orcid.org/0000-0002-2691-5503

\section{References}

1. Driver JA. Inverse association between cancer and neurodegenerative disease: review of the epidemiologic and biological evidence. Biogerontol. 2014;15(6):547-57.

2. Houck AL, Seddighi S, Driver JA. At the crossroads between neurodegeneration and cancer: a review of overlapping biology and its implications. Current aging science. 2018;11:77-89.

3. Du L, Pertsemlidis A. Cancer and neurodegenerative disorders: pathogenic convergence through microRNA regulation. J Mol Cell Biol. 2011;3(3):176-180.

4. Guang MHZ, Kavanagh EL, Dunne LP, Dowling P, Zhang L, Lindsay S, et al. Targeting proteotoxic stress in cancer: a review of the role that protein quality control pathways play in oncogenesis. Cancers. 2019;11:66.

5. Wang M, Kaufman RJ. The impact of the endoplasmic reticulum protein-folding environment on cancer development. Nat Rev Cancer. 2014;14:581-97.

6. Kanatsuka A, Kou S, Makino H. IAPP/amylin and b-cell failure: implication of the risk factors of type 2 diabetes. Diabetol Int. 2018;9:143-157.

7. Mukherjee A, Morales-Scheihing D, Butler PC, Soto C. Type 2 diabetes as a protein misfolding disease. Trends Mol Med. 2015;21(7):439-49. 
8. Barbagallo M, Dominguez LJ. Type 2 diabetes mellitus and Alzheimer's disease. World J. Diabetes. 2014;5:889-93.

9. Irie F, Fitzpatrick AL, Lopez OL, Kuller LH, Peila R, Newman AB, et al. Enhanced risk for Alzheimer disease in persons with type 2 diabetes and APOE epsilon4: the Cardiovascular Health Study Cognition Study. Arch Neurol. 2008;65(1):89-93.

10. Larsson SC, Mantzoros CS, Wolk A. Diabetes mellitus and risk of breast cancer: a meta-analysis. Int J Cancer. 2007;121(4):856-62.

11. Larsson SC, Orsini N, Wolk A. Diabetes mellitus and risk of colorectal cancer: a meta-analysis. J Nation Cancer Instit. 2005;97(22):1679-87.

12. Friberg E, Orsini N, Mantzoros CS, Wolk A. Diabetes mellitus and risk of endometrial cancer: a metaanalysis. Diabetologia. 2007;50(7):1365-74.

13. Huxley R, Ansary-Moghaddam A, Berrington de González A, Barzi F, Woodward M. Type-ll diabetes and pancreatic cancer: a meta-analysis of 36 studies. Br J Cancer. 2005;92(11):2076-83.

14. El-Serag HB, Hampel H, Javadi F. The association between diabetes and hepatocellular carcinoma: a systematic review of epidemiologic evidence. Clin Gastroentol Hepatol. 2006:4(3):369-80.

15. Fang H, Yao B, Yan $Y, X u H$, Liu Y, Tang $H$, et al. Diabetes mellitus increases the risk of bladder cancer: an updated meta-analysis of observational studies. Diabetes Technol Ther. 2013;15(11):914-22.

16. Shikata K, Ninomiya T, Kiyohara Y. Diabetes mellitus and cancer risk: review of the epidemiological evidence. Cancer Sci. 2013;104:9-14.

17. Giovannucci E, Harlan DM, Archer MC, Bergenstal RM, Gapstur SM, Habel LA, et al. Diabetes and cancer: a consensus report. CA Cancer J Clin. 2010;60:207-21.

18. Driver JA, Beiser A, Au R, Kreger BE, Splansky GL, Kurth T, et al. Inverse association between cancer and Alzheimer's disease: results from the Framingham Heart Study. BMJ. 2012;344:e1442.

19. Roe CM, Behrens MI, Xiong C, Miller JP, Morris JC. Alzheimer disease and cancer. Neurology. 2005;64:895-98.

20. Roe CM, Fitzpatrick AL, Xiong C, Sieh W, Kuller L, Miller JP, et al. Cancer linked to Alzheimer disease but not vascular dementia. Neurology. 2010;74:106-12.

21. Feng DD, Cai W, Che X. The associations between Parkinson's disease and cancer: the plot thickens. Transl Neurodegener. 2015;4:20.

22. Bajaj A, Driver JA, Schernhammer ES. Parkinson's disease and cancer risk: a systematic review and meta-analysis. Cancer Causes Control. 2010;21:697-707.

23. Lin PY, Chang SN, Hsiao TH, Huang BT, Lin CH, Yang PC. Association between Parkinson disease and risk of cancer in Taiwan. Jama Oncol. 2015;1:633-640.

24. Chun C, Kim S, Lee J, Lee S. Republic of Korea. Health system review. Health Syst Transit. 2009;11;1-184.

25. Lee J, Lee JS, Park SH, Shin SA, Kim K. Cohort profile: the National Health Insurance Service-National Sample Cohort (NHIS-NSC), South Korea. Int J Epidemiol. 2017;46(2):e15. 
26. Shin DW, Cho B, Guallar E. Korean National Health Insurance database. JAMA Intern Med. 2016;176(1):138.

27. Ahn E. Introducing big data analysis using data from National Health Insurance Service. Korean J Anesthesiol. 2020;73(3):205-11.

28. Song SO, Jung CH, Song YD, Park CY, Kwon HS, Cha BS, et al. Background and data configuration process of a nationwide population-based study using the korean national health insurance system. Diabetes Metab J. 2014;38:395-403.

29. StataCorp. Stata Statistical Software. Release 16. College Station, Texas: StataCorp LLC; 2019.

30. Seo J, Park M. Molecular crosstalk between cancer and neurodegenerative diseases. Cell Mol Life Sci. 2020;77(14):2659-80.

31. Angelucci F, Hort J. Prolyl isomerase Pin1 and neurotrophins: a loop that may determine the fate of cells in cancer and neurodegeneration. Ther Adv Med Oncol. 2017;9(1):59-62.

32. Harris CC, Hollstein M. Clinical implications of the p53 tumor-suppressor gene. N Engl J Med. 1993;329(18):1318-27.

33. Navalkar A, Ghosh S, Pandey S, Paul A, Datta D, Maji SK. Prion-like p53 Amyloids in Cancer. Biochemistry. 2020;59(2):146-55.

34. Gong H, Yang X, Zhao Y, Petersen RB, Liu X, Liu Y, et al. Amyloidogenicity of p53: a hidden link between protein misfolding and cancer. Curr Protein Pept Sci. 2014;16(2):135-46.

35. Rangel LP, Costa DC, Vieira TC, Silva JL. The aggregation of mutant p53 produces prion-like properties in cancer. Prion. 2014;8(1):75-84.

36. de Oliveira GA, Rangel LP, Costa DC, Silva JL. Misfolding, aggregation, and disordered segments in cAbl and p53 in human cancer. Front Oncol. 2015;5:97.

37. Hieronymus L, Griffin S: Role of amylin in type 1 and type 2 diabetes. Diabetes Educ. 2015;41(1 Suppl):47S-56S.

38. Bharadwaj P, Wijesekara N, Liyanapathirana M, Newsholme P, Ittner L, Fraser P, et al. The link between type 2 diabetes and neurodegeneration: roles for amyloid- $\beta$, amylin, and tau proteins. $J$ Alzheimers Dis. 2017;59(2):421-32.

39. Martinez-Valbuena I, Valenti-Azcarate R, Amat-Villegas I, Riverol M, Marcilla I, de Andrea CE, et al. Amylin as a potential link between type 2 diabetes and alzheimer disease. Ann. Neurol. 2019;86:539-51.

40. Seshasai SRK, Kaptoge S, Thompson A, Angelantonio ED, Gao P, Sarwar N, et al. Diabetes mellitus, fasting glucose, and risk of cause-specific death. N Engl J Med. 2011;364(9):829-41.

41. Johnson JA, Carstensen B, Witte D, Bowker SL, Lipscombe L, Renehan AG on behalf of the Diabetes and Cancer Research Consortium-Show fewer authors. Diabetes and cancer (1): evaluating the temporal relationship between type 2 diabetes and cancer incidence. Diabetologia. 2012;55:160718. 
42. Yang WS, Shu XO, Gao J, Li HL, Cai H, Yang G, et al. Prospective evaluation of type 2 diabetes mellitus on the risk of primary liver cancer in Chinese men and women. Ann Oncol. 2013;24:167985.

43. Handelsman Y, Leroith D, Bloomgarden ZT, Dagogo-Jack S, Einhorn D, Garber AJ, et al. Diabetes and cancer-an AACE/ACE consensus statement. Endocr Pract. 2013;19(4):675-93.

44. Vigneri P, Frasca F, Sciacca L, Pandini G, Vigneri R. Diabetes and cancer. Endocr Relat Cancer. 2009;16(4):1103-23.

\section{Figures}

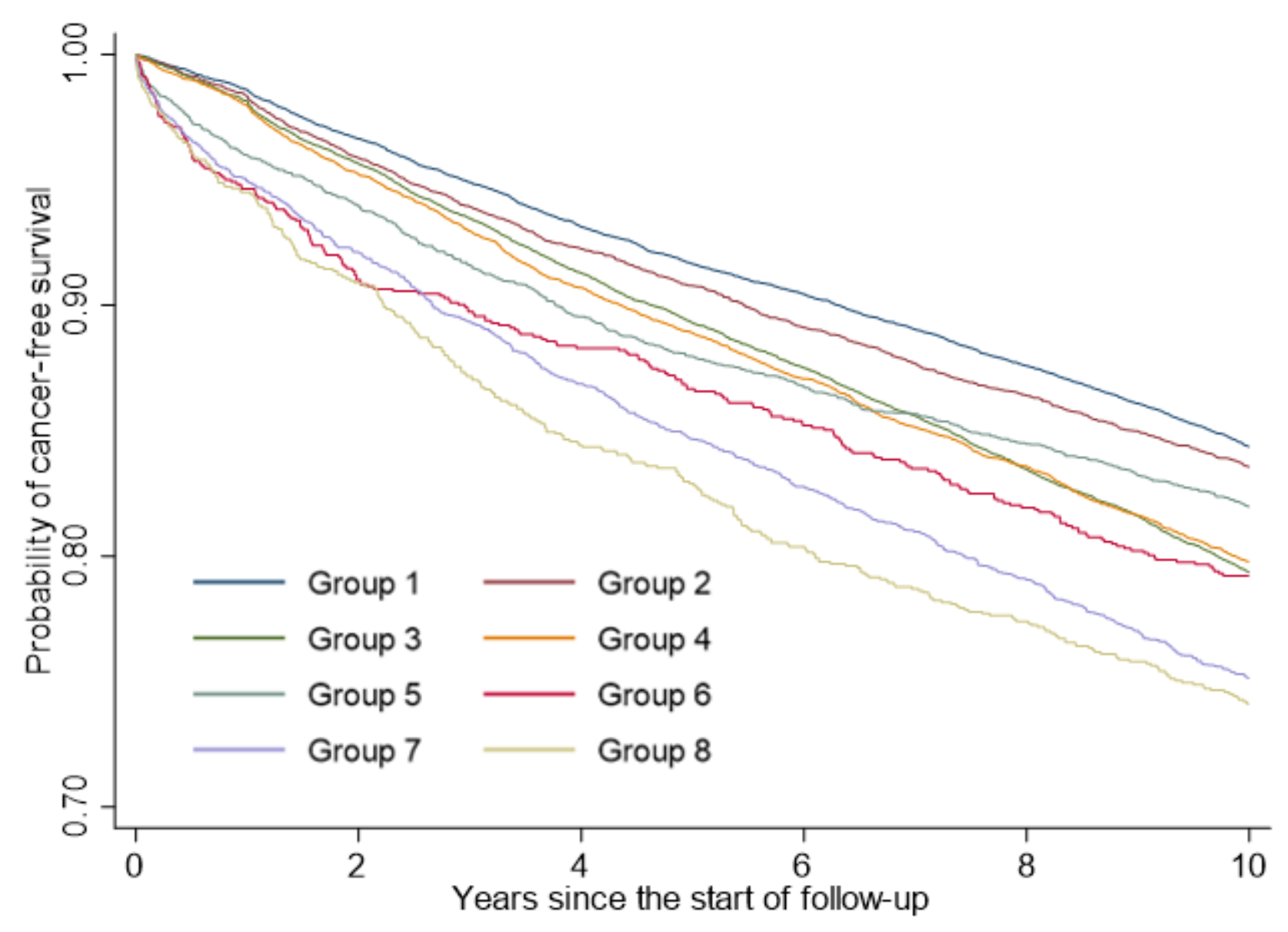

\section{Figure 1}

Kaplan-Meier survival curves for cancer-free survival Group 1 is a control group without both newly diagnosed ND and T2D ( $n=14,825,26.5 \%)$, Group 2 is a control group with only newly diagnosed ND (no T2D) ( $n=6,222,11.1 \%$ ), Group 3 is a control group with only newly diagnosed T2D (no ND) ( $n=16,482$, $29.4 \%)$, Group 4 is a control group with both newly diagnosed ND and T2D ( $n=9,213,16.4 \%)$, Group 5 is an ND group without both newly diagnosed ND and T2D ( $n=3,499,6.2 \%)$, Group 6 is an ND group with only newly diagnosed ND (no T2D) ( $n=1,102,2.0 \%$ ), Group 7 is an ND group with only newly diagnosed 
T2D (no ND) ( $\mathrm{n}=3,381,6.0 \%)$, Group 8 is an ND group with both newly diagnosed ND and T2D $(\mathrm{n}=$ $1,310,2.3 \%)$ 